\title{
A practical examination of the errors arising in the direct collocation boundary element method for acoustic scattering
}

\author{
B.E. Treeby ${ }^{\mathrm{a}, *, 1}, \mathrm{~J}$. Pan $^{\mathrm{b}}$ \\ ${ }^{a}$ Department of Medical Physics and Bioengineering, University College London, Gower Street, London WC1E 6BT, United Kingdom \\ ${ }^{\mathrm{b}}$ School of Mechanical Engineering, The University of Western Australia, 35 Stirling Highway, Crawley, WA 6009, Australia
}

\section{A R T I C L E I N F O}

\section{Article history:}

Received 2 December 2008

Accepted 25 June 2009

Available online 25 July 2009

Keywords:

Boundary element method

Non-linear coordinate transforms

Singular integrals

Integration error

Boundary singularities

\begin{abstract}
A B S T R A C T
For many engineers and acousticians, the boundary element method (BEM) provides an invaluable tool in the analysis of complex problems. It is particularly well suited for the examination of acoustical problems within large domains. Unsurprisingly, the widespread application of the BEM continues to produce an academic interest in the methodology. New algorithms and techniques are still being proposed, to extend the functionality of the BEM, and to compute the required numerical tasks with greater accuracy and efficiency. However, for a given global error constraint, the actual computational accuracy that is required from the various numerical procedures is not often discussed. Within this context, this paper presents an investigation into the discretisation and computational errors that arise in the BEM for acoustic scattering. First, accurate routines to compute regular, weakly singular, and nearly weakly singular integral kernels are examined. These are then used to illustrate the effect of the requisite boundary discretisation on the global error. The effects of geometric and impedance singularities are also considered. Subsequently, the actual integration accuracy required to maintain a given global error constraint is established. Several regular and irregular scattering examples are investigated, and empirical parameter guidelines are provided.
\end{abstract}

(c) 2009 Elsevier Ltd. All rights reserved.

\section{Introduction}

The numerical solution of boundary integral equations via the boundary element method (BEM) has become a popular approach for solving acoustical problems. The now ubiquitous presence of high-speed computing has made it possible for engineers and acousticians to investigate any number of complex scenarios for which analytical solutions do not exist, or for which approximate solutions are not well suited. Unsurprisingly, the widespread application of the BEM continues to produce an academic interest in the methodology. New algorithms and techniques are still being proposed which extend the functionality of the BEM and allow the required numerical tasks to be computed with greater accuracy and efficiency. There are now a myriad of approaches for formulating the governing equations, discretising and computing the boundary quantities, computing singular integrals, and ensuring the uniqueness of the final solution. However, the overwhelming extent of the available literature paradoxically increases the difficulty of understanding the technique. Much of

\footnotetext{
* Corresponding author. Tel.: +44207679 7086; fax: +44207679 0255 .

E-mail address: btreeby@mpb.ucl.ac.uk (B.E. Treeby).

1 This work was completed while at the School of Mechanical Engineering, The University of Western Australia.
}

the advanced literature is focused on very specific aspects of the BEM and the consequence of utilising particular numerical routines or element types on the overall problem accuracy is not always immediately clear.

In an academic sense, formal mathematical investigations on the convergence of the various discretisation and numerical procedures lie within the (now) historical literature (e.g., [1,2]). In contrast, within engineering fields it is recurrently only the relative number of elements that is considered, with six elements per wavelength the most frequently prescribed guideline (see [3] and references therein). In fact, the errors in the BEM can arise from several distinct locations:

- the discrete approximation of the varying boundary quantities;

- the discrete approximation of the boundary geometry;

- geometric and impedance singularities;

- the numerical computation of regular integrals;

- the numerical computation of singular integrals;

- and, the solution of the system of equations.

The first three may be regarded as discretisation errors, the next two as quadrature errors, and the last incorporates the wellknown non-uniqueness difficulty. Despite the continual advances in numerical and BEM literature, the actual accuracy that is 
required from the various computations to maintain a given level of global accuracy is rarely discussed. In this context, these errors are explored in detail here, primarily in relation to the direct collocation BEM for the two-dimensional (2D) Helmholtz equation. The investigation is practical in nature, rather than mathematically formal, and empirical guidelines are established for the various error constituents.

The basic formulation and use of the collocation BEM is well known (e.g., [4-7]). Arbitrary boundary surfaces are first discretised into a finite number of boundary elements. Across each element, approximations are made about the variation of the boundary quantities (the pressure and velocity in the direct BEM) and geometry. The boundary quantities are assigned at a discrete number of nodes per element (referred to as the element order), and shape functions are used to approximate the values at the other positions. The variation of the boundary geometry is modelled in the same way. The overall computational effort is directly related to the total number of boundary nodes (often referred to as the number of system degrees of freedom).

Considering first only the error associated with the discretised approximation of the varying boundary quantities, the solution convergence with various mesh modifications is well documented (e.g., [8]). If the number of elements is increased without modifying the number of nodes per element (the so-called refinement or h-method), an algebraic convergence related to the element order is obtained as the total number of degrees of freedom is increased. If the number of elements is kept constant and instead the number of nodes per element is increased (the socalled enrichment or p-method), an exponential convergence is obtained. However, if the boundary is not smooth, i.e., it contains geometric or impedance singularities, the convergence of the p-method degenerates. Boundary singularities occur because of abrupt changes or discontinuities in the surface profile or its properties. These cause the solution and its derivatives to vary rapidly or become unbounded [9]. A combination of the refinement method near boundary singularities and the enrichment method elsewhere (the so-called hp-method) can restore the exponential convergence of the $\mathrm{p}$-method $[10,11]$.

In addition to the number of elements (and nodes per element), the continuity of the quantities around the boundary, enforced by the element type, is also of importance. If the outer nodes on each element are placed at the endpoints and shared between adjoining elements, the surface quantities remain continuous around the boundary. Continuous isoparametric linear and parabolic elements are the most widely used in engineering applications [3]. Alternatively, if the outer nodes are located within the element, the boundary quantities become discontinuous. Compared to continuous elements, this requires an increase in the overall number of nodes to describe the same order of boundary variation (as nodes are no longer shared between elements). Despite this, if the nodes are positioned correctly, discontinuous elements can provide an increase in accuracy for the same number of system degrees of freedom [12]. For threedimensional quadrilateral or triangular elements, the zeros of the Legendre polynomials provide this optimum position. Discontinuous elements also have the distinct advantage of the normal direction always being well defined. Similarly, they naturally satisfy the $C^{1}$ continuity condition.

In contrast to the approximation of the boundary quantities, the variation of the boundary geometry should remain continuous from one element to the next. For discontinuous boundary quantities, this requires the use of continuous shape functions based on a different set of nodal values. However, this does not add any particular computational complexity, as the geometric coordinates of the utilised nodes are required in any case. Rather, the inherent separation of the boundary geometry and quantities makes it straightforward to use non-isoparametric elements. This is computationally efficient when using higher-order shape functions to describe the variation of the boundary quantities for simple boundary geometries. The requirement for the geometric discretisation is that the variation of the boundary elements matches that of the underlying problem. For isoparametric linear elements, the error from the geometric discretisation of curved boundaries is of the same order as that from the discretisation of the boundary quantities [8].

Returning to the convergence of the BEM, rather than simply uniformly increasing the total number of elements or nodes to improve accuracy, local error indicators can be used to specify regions where the boundary discretisation must be refined [13]. The use of suitable adaptive methods can significantly improve the rate of convergence, as degrees of freedom automatically become concentrated near boundary singularities and other areas where the surface quantities vary rapidly [13-16]. However, the computation of many error indicators (upon which the adaptive procedures rely) is very expensive; on the order of the BEM analysis itself [17]. Moreover, at each step of the mesh refinement, the problem must be recomputed. As an alternative, boundary meshes may be graded a priori so that the optimal values of convergence are maintained $[11,18]$. Similarly, boundary singularities may also be counteracted by using specially designed interpolation functions [8].

Apart from discretisation errors, the computation of the element-wise boundary integration (typically via numerical quadrature) may also introduce errors into the BEM. In a commercial BEM package, the modification of the quadrature routines is unlikely to be facilitated. Moreover, in classical studies, the quadrature error is rarely considered important in comparison with that from the boundary discretisation (e.g., [19]). However, care must be taken that it is sufficiently small [20]. For regular integrals, this constraint is of no particular consequence as standard quadrature routines are able to compute the required integration tasks very accurately. Nonetheless, it is important to ensure that singular and nearly singular integrals can be computed with the same accuracy. This is particularly important for boundaries that yield coefficient matrices where the diagonal (singular) terms are dominant. Although integration errors can generally be reduced by increasing the order of the quadrature routines, doing so arbitrarily without consideration of the actual accuracy requirements can result in a significant computational penalty.

The final source of error relates to the solution of the system of equations for the unknown boundary quantities. For a wellconditioned coefficient matrix, this error is negligible. However, at certain characteristic frequencies that depend on the problem geometry, the solution may no longer be unique. This nonuniqueness has no physical analogy and is simply a mathematical artefact of the integral formation; the objective problem itself has a unique solution. These (so-called) characteristic frequencies occur at the resonant frequencies of the corresponding interior Dirichlet problem (the non-uniqueness problem occurs because the interior and exterior operators are adjoint [21]). The most mathematically robust way to overcome this difficulty is to use the Helmholtz integral equation in combination with its normal derivative. The latter conversely suffers from non-uniqueness at the resonant frequencies of the interior Neumann problem. Burton and Miller showed that the two integral equations share only one common solution and thus their combination will overcome the mathematical uniqueness difficulty [22]. However, the normal derivative integral equation contains a hyper-singular integral kernel which is numerically formidable (the integral does not exist because of divergent terms in the primitive function of the 
integrand [23]). A large body of literature exists on the regularisation and computation of these integrals, which remains a somewhat problematic task [24-26].

The non-uniqueness problem may also be overcome via the combined Helmholtz integral equation formulation (CHIEF) proposed by Schenck [27]. Here, a small additional set of equations are formulated by collocating the evaluation point at a series of so-called 'CHIEF points' from the interior of the scattering body. These additional constraints provide an overdetermined system of equations that is typically better conditioned. The success of the CHIEF method is reliant on avoiding interference between the selected internal CHIEF points and the nodal surface of the interior Dirichlet eigen-functions. If a CHIEF point falls on this nodal surface, the additional equation does not provide a linearly independent constraint. Generally only a small number of 'effective' CHIEF points are needed [28-31]. However, the density of the interior nodal surfaces increases with wavenumber and for a scatterer of arbitrary geometry the location of these surfaces is not known a priori. The inherent difficulty in the CHIEF method is thus the systematic selection of CHIEF points, although, several methods for improving the robustness of the CHIEF method have been proposed [32-35].

The purpose of the current paper is to provide a practical and contiguous analysis of the various sources of error in the solution of acoustic scattering problems using the BEM. The analysis is not mathematically formal in nature, but rather is designed to give BEM users a functional indication of factors affecting the computational accuracy. In particular, the accuracy of the various numerical procedures that is required to maintain a given global error bound is considered. In Section 2, a brief overview of the numerical implementation of the direct collocation BEM for the 2D Helmholtz equation is given. This provides the context for later discussion. While the integral equations may be derived using other formulations (e.g., the indirect BEM), and the boundary quantities solved using other weighted residuals (e.g., the Galerkin method), the direct collocation BEM remains a popular approach for acoustical problems. For example, this formulation has particular relevance to the design of acoustic barriers (e.g., [36,37]); a familiar problem in acoustics. In Section 3, the element-wise convergence of numerical quadrature routines is examined. Both regular, weakly singular, and nearly weakly singular integral kernels are considered. In Section 4, the global effect of the boundary discretisation is examined using a cylindrical scatterer as an example. This discussion is extended in Section 5 to include the effect of boundary singularities. In Section 6, the accuracy of the integration routines required to maintain a given global error bound is established. These results are then summarised into a set of empirical guidelines and applied to a final scattering example. Discussion and summary are given in Section 7.

Finally, before beginning the analysis, it is appropriate to briefly discuss the expectation of accuracy required for engineering problems. While a particular numerical solution may have converged to a high degree of accuracy, it is unlikely many of the classical acoustic assumptions (uniform boundary conditions, a non-refracting atmosphere, etc.) are unequivocally met by the corresponding physical situation. In this regard, it is not appropriate to recommend parameter guidelines that guarantee the absolute convergence of the numerical problem when the physical counterpart may contain uncertainties of a much higher percentage. On the other hand, numerical methods are often used to compare subtle design and scenario variations. In this case, large error values make it difficult to quantify the effect of these changes. Considering these factors, here a global BEM error requirement of $\sim 1 \%$ will be regarded as appropriate.

\section{Numerical implementation of the direct collocation method for the Helmholtz equation}

\subsection{Formulation of the boundary integral equations}

For small amplitude time-harmonic disturbances of a homogeneous and inviscid fluid, the spatially dependent excess pressure $p$ is governed by the Helmholtz equation $\nabla^{2} p+k^{2} p=0$. Here $k$ is the wave-number, and a harmonic time component of the form $e^{-i \omega t}$ has been assumed. The subsequent formulation of the Helmholtz integral equation using Green's second identity for the harmonic operator (based on the Gauss-Green theorem) is well known (e.g., [21]) and may be expressed as

$$
\begin{aligned}
& p_{\text {in }}(P)+\int_{S}\left(p(Q) \frac{\partial G(Q \mid P)}{\partial n_{Q}}-G(Q \mid P) \frac{\partial p(Q)}{\partial n_{Q}}\right) d S \\
& \quad= \begin{cases}p(P) & , P \in V \\
\frac{1}{2} p(P) & , P \in S \\
0 & , P \notin(V \cup S)\end{cases}
\end{aligned}
$$

where $p_{i n}(P)$ is a source term, $P$ the evaluation point, $Q$ an integration point on the boundary surface $S$, and $G(Q \mid P)$ the fundamental solution (Green's function) for the acoustic domain of interest. The solution for $P \in S$ assumes the variation of the boundary is smooth which always holds at the nodal locations for discontinuous elements. In 2D free-space, the Green's function is an outgoing cylindrical wave given by $G(Q \mid P)=(i / 4) \mathrm{H}_{0}^{(1)}\left(k r_{Q P}\right)$ and represents the effect experienced at $P$ of a source at $Q$ radiating into the domain (or vice versa). Here $r_{Q P}=\left|\mathbf{r}_{Q}-\mathbf{r}_{P}\right|$, and $H_{0}^{(1)}$ is the Hankel function of the first kind of zero order. The source term in 2D free-space is similarly given by $p_{i n}(P)=P_{i n} H_{0}^{(1)}\left(k r_{S P}\right)$, where $r_{S P}$ is the separation radius between the source and evaluation positions, and $P_{\text {in }}$ is the source strength.

To reduce the number of unknown boundary quantities, the Helmholtz integral equation is generally reformulated using a surface boundary condition relating $\partial p / \partial n$ to $p$. For acoustic scattering problems, the Robin boundary condition is of most interest

$\frac{\partial p}{\partial n}+i k \beta p=g$

and applies to the boundary of the scattering surface $S$ within the acoustic domain $V$. Here $n$ is the normal vector of the boundary pointing into the domain, and $\beta$ the specific acoustic admittance [see Fig. 1]. The function $g$ is zero for stationary scattering problems (passive boundary conditions) and non-zero for radiation problems (active boundary conditions). When $\beta=0$, Eq. (2) degenerates to a Neumann boundary condition.

\subsection{Boundary collocation}

Unless the acoustic quantities on the boundary surface are already explicitly known, Eq. (1) cannot immediately be used to solve for the pressure within the domain; these quantities must first be calculated. To proceed, assumptions must be made about the variation of the boundary quantities over each element. In the collocation approach, the boundary quantities are assigned values at a discrete number of nodal locations per element with the remaining variation modelled using shape functions. If $f$ is the varying quantity, the local element coordinates are $-1 \leq \xi \leq 1$, and the order of the boundary variation for $f$ is $\alpha_{\max }$ (i.e., there are $\alpha=1,2, \ldots, \alpha_{\max }$ nodes per element), the variation of $f$ over each 

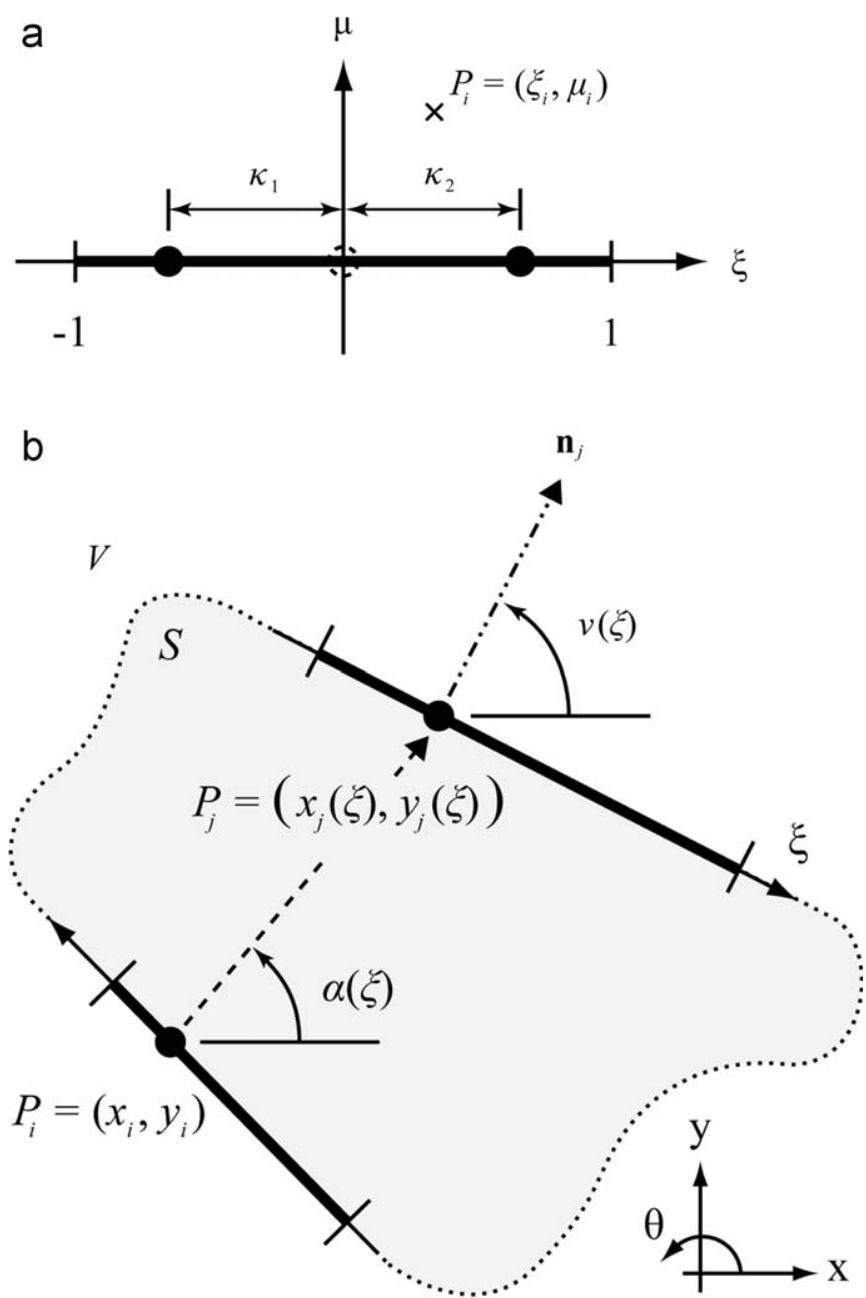

Fig. 1. (a) Local element coordinates showing the node placement for discontinuous elements and the evaluation point $P_{i}$, and (b) an arbitrary scattering body showing the mapping between global and local coordinates, and the element normals.

element can be written as

$f(\xi)=\sum_{\alpha}^{\alpha_{\max }} \psi_{\alpha}(\xi) f_{\alpha}$,

where $\psi_{\alpha}(\xi)$ and $f_{\alpha}$ represent the shape function and the discrete quantity value at the $\alpha$ th node on the element, respectively. The quantity $f$ may be either the varying pressure $p(\xi)$ on the boundary, or the geometric variables $x(\xi)$ and $y(\xi)$.

To find the pressure on the boundary surface, the evaluation point $P$ is then collocated at each of the boundary nodes $P_{i}$ (where $\left.i=1,2, \ldots, i_{\max }\right)$. Using Eq. (2), Eq. (1) now becomes

$$
\begin{aligned}
& p_{\text {in }}\left(P_{i}\right)+\sum_{j} \int_{S_{j}} p\left(P_{j}\right)\left(\frac{\partial G\left(r_{j i}\right)}{\partial n_{j}}+i k \beta_{j} G\left(r_{j i}\right)\right) d S-\sum_{j} \int_{S_{j}} g_{j} G\left(r_{j i}\right) d S \\
& =\frac{1}{2} p\left(P_{i}\right),
\end{aligned}
$$

where $p\left(P_{i}\right)$ represents the pressure at the $i$ th boundary node, the integration point $Q$ has been replaced with $P_{j}$, and $p\left(P_{j}\right)$ represents the pressure across the $j$ th surface element $S_{j}$ (where $j=1$, $\left.2, \ldots, j_{\max }\right)$. This is related to the discrete nodal pressure values using Eq. (3) [see Fig. 1(b)]. Note, the lower case $i$ not used as a subscript represents the imaginary unit.
Momentarily disregarding numerical singularities (which occur when $r_{j i} \rightarrow 0$ ), the integrals within Eq. (4) are computed using numerical quadrature. For regular Gaussian quadrature, this gives

$I=\int_{-1}^{1} f(\xi) J(\xi) d \xi=\sum_{\chi=1}^{K} w_{\chi} f\left(\xi_{\chi}\right) J\left(\xi_{\chi}\right)$

where $K$ is the number of Gauss (integration) points, $w_{\chi}$ and $\xi_{\chi}$ the weights and abscissas of the Gaussian quadrature of order $\chi$, and $J(\xi)$ represents the transformation Jacobian that maps the global coordinate system onto the local quadrature coordinate system $-1 \leq \xi \leq 1$. The Gaussian weights and abscissas can be computed using Legendre's orthogonal polynomials [38], or alternatively are tabulated to a high degree of accuracy in many standard textbooks. The transformation Jacobian is given by

$J(\xi)=\sqrt{\left(\frac{d x}{d \xi}\right)^{2}+\left(\frac{d y}{d \xi}\right)^{2}}$,

where $x(\xi)$ and $y(\xi)$ are also given by Eq. (3) using the continuous shape functions of the required order.

Expanding the element-wise approximation of the boundary quantities, the pressure on all $i_{\max }$ nodes can then be solved using the system of equations $[(1 / 2) \mathbf{I}-\mathbf{A}]\{\mathbf{p}\}=\{\mathbf{B}\}$, where $\mathbf{p}$ is a vector of the unknown pressure at each boundary node, $\mathbf{I}$ the identity matrix, the coefficient matrix $\mathbf{A}$ is square and given by

$$
\begin{aligned}
A_{i, j \alpha}= & \int_{-1}^{1} \frac{\partial G\left(r_{j i}(\xi)\right)}{\partial n_{j}} \psi_{\alpha}(\xi) J(\xi) d \xi \\
& +i k \beta_{j} \int_{-1}^{1} G\left(r_{j i}(\xi)\right) \psi_{\alpha}(\xi) J(\xi) d \xi,
\end{aligned}
$$

and $\mathbf{B}$ is a source vector given by

$B_{i}=p_{i n}\left(P_{i}\right)-\sum_{j} \sum_{\alpha} g_{j} \int_{-1}^{1} G\left(r_{j i}(\xi)\right) \psi_{\alpha}(\xi) J(\xi) d \xi$

Here $\beta_{j}$ and $g_{j}$ are the boundary condition parameters on the $j$ th element; recall there are $i_{\max }$ nodes in total made up from $j_{\max }$ elements each with $\alpha_{\max }$ nodes where the pressure is assigned. As the element-wise approximation of the boundary geometry within the integral kernels in Eqs. (7) and (8) is independent from that of the pressure [i.e., the computation of the geometric terms $r_{j i}(\xi)$ and $J(\xi)$ using Eq. (3) is not dependent on the $\psi_{\alpha}(\xi)$ and $\alpha_{\max }$ chosen to compute the boundary pressure], it is straightforward to implement non-isoparametric elements.

The first integral term of Eq. (7) requires the normal derivative of the Green's function. This is computed by

$\frac{\partial G\left(r_{j i}(\xi)\right)}{\partial n_{j}}=\frac{\partial G\left(r_{j i}(\xi)\right)}{\partial r_{j i}} \frac{\partial r_{j i}(\xi)}{\partial n_{j}}$,

where the radial derivative of the 2D free-space Green's function is given by

$\frac{\partial G\left(r_{j i}(\xi)\right)}{\partial r_{j i}}=-\frac{i k}{4} H_{1}^{(1)}\left(k r_{j i}(\xi)\right)$,

the radial variable is computed using

$r_{j i}(\xi)=\sqrt{\left(x_{j}(\xi)-x_{i}\right)^{2}+\left(y_{j}(\xi)-y_{i}\right)^{2}}$,

and the directional derivative is calculated by [see Fig. 1(b)]

$\frac{\partial r_{j i}(\xi)}{\partial n_{j}}=\nabla r_{j i}(\xi) \cdot \mathbf{n}_{\mathbf{j}}=\cos (v(\xi)-\alpha(\xi))$. 
Using the gradient of the geometry at $\xi$, the required angles $\alpha$ and $v$ are computed by

$\alpha(\xi)=a \tan 2\left(y_{j}(\xi)-y_{i}, x_{j}(\xi)-x_{i}\right)$

$v(\xi)=a \tan 2\left(\frac{d y_{j}(\xi)}{d \xi}, \frac{d x_{j}(\xi)}{d \xi}\right)+\frac{\pi}{2}$.

Once the pressure on the boundary has been calculated, Eq. (1) may then be directly utilised to calculate the pressure at any location within the domain. Note, in the examples given in the following sections, only passive Robin and Neumann boundary conditions are used [i.e., $g=0$ and $B_{i}=p_{i n}\left(P_{i}\right)$ ]. While not directly considered, the influence of the additional integral term in Eq. (8) can be inferred from the discussion of the analogous term within Eq. (7). The results for Dirichlet boundary conditions may be similarly deduced.

\subsection{Discontinuous shape functions}

For the BEM examples presented here, discontinuous elements were used as given in Ref. [7]. For constant elements, $\alpha_{\max }=1$ and the shape function is simply $\psi_{1}(\xi)=1$ (the pressure is constant across the element). For linear elements, $\alpha_{\max }=2$ and the shape functions are given by

$\psi_{1}(\xi)=\frac{1}{\kappa_{1}+\kappa_{2}}\left(\kappa_{2}-\xi\right)$
$\psi_{2}(\xi)=\frac{1}{\kappa_{1}+\kappa_{2}}\left(\kappa_{1}+\xi\right)$.

Similarly, for parabolic (quadratic) elements, $\alpha_{\max }=3$ and the shape functions are given by

$\psi_{1}(\xi)=\frac{\kappa_{2}}{\bar{\kappa}}\left(\xi-\kappa_{2}\right) \xi$

$\psi_{2}(\xi)=\frac{\kappa_{1}+\kappa_{2}}{\bar{\kappa}}\left[\kappa_{1} \kappa_{2}+\left(\kappa_{2}-\kappa_{1}\right) \xi-\xi^{2}\right]$

$\psi_{3}(\xi)=\frac{\kappa_{1}}{\bar{\kappa}}\left(\xi+\kappa_{1}\right) \xi$

In Eqs. (14) and (15), $\kappa_{1}$ and $\kappa_{2}$ represent the offset of the outer nodal points from the element origin (with $0<\kappa_{1}, \kappa_{2} \leq 1$ ) [see Fig. 1(a)]. For parabolic elements, the central node is assumed to be located at $\xi=0$, and $\bar{\kappa}=\kappa_{1} \kappa_{2}\left(\kappa_{1}+\kappa_{2}\right)$. The optimum values of $\kappa_{1}$ and $\kappa_{2}$ are given by the zeros of Legendre polynomials [12]. This yields $\kappa_{1,2}=0.5774$ for linear and $\kappa_{1,2}=0.7746$ for parabolic boundary variations (a similar analysis to that presented in Ref. [12] was also used here to confirm these as the optimum nodal locations for one-dimensional elements). If the boundary nodes are placed at the extremities (i.e., $\kappa_{1,2}=1$ ), Eqs. (14) and (15) yield the continuous shape functions discussed by many authors.

\subsection{Overcoming the non-uniqueness problem}

For the BEM examples presented here, the non-uniqueness problem was overcome using an extension of the CHIEF method (described herein) [35]. Along with the boundary, a small number of representative interior coordinates are discretised. A larger array of potential CHIEF points is then derived by linearly interpolating between these coordinates. The system of equations is first solved without any CHIEF points, and the uniqueness of the solution monitored using the matrix condition number [39]. If a large condition number is detected, the solution at each of the
CHIEF points is computed. The best CHIEF points are then selected based on the maximum deviation of the pressure from zero. This eliminates points that lie on an interior nodal surface. The system of equations becomes

$\left[\begin{array}{l}\frac{1}{2} \mathbf{I}-\mathbf{A} \\ 0-\mathbf{A}_{\text {Chief }}\end{array}\right]\{\mathbf{p}\}=\left\{\begin{array}{l}\mathbf{B} \\ \mathbf{B}_{\text {Chief }}\end{array}\right\}$,

and is re-solved using a Lagrange or least squares procedure. Here $\mathbf{A}_{\text {Chief }}$ and $\mathbf{B}_{\text {Chief }}$ are given by Eqs. (7) and (8), respectively, with the $i$ th element node replaced by the $n$th CHIEF point. As the primary computational effort in the BEM is associated with the filling of the coefficient matrices (and not their solution), this approach does not significantly increase the total computation time. Moreover, as no additional functions are required for the evaluation of the CHIEF terms, it is simple to implement. For the analyses presented here, this approach worked robustly. From this point it is assumed that the non-uniqueness problem has been adequately addressed; no further discussion is given.

\section{Element-wise convergence of numerical quadrature routines}

\subsection{Computation of regular integrals}

Before beginning to examine the effect of the boundary discretisation on the overall convergence of objective problems, it is constructive to revisit the local convergence of the elementwise numerical integration. For regular integrals, the superiority of Gaussian type quadrature routines over other numerical integration techniques is well established (e.g., [8]). With $K$ Gauss-points, Gaussian quadrature is able to exactly integrate polynomials of up to order $2 K-1$. To illustrate the effect of the number of Gauss-points on integration accuracy, a simplification of the second integral in Eq. (7) is taken [see Fig. 1(a)]. Here the point $P_{i}$ is assumed to be within the local domain at $\left(\xi_{i}, \mu_{i}\right)$ and the integration is performed on the $\xi$-axis over the interval $[-1,1]$. This yields the following element-wise integration

$I=\int_{-1}^{1} H_{0}^{(1)}\left(k \sqrt{\left(\xi-\xi_{i}\right)^{2}+\mu_{i}^{2}}\right) d \xi$.

The length of the integration element in local coordinates is 2, and the wave-number $k$ is set to 1 . This corresponds to approximately 3 elements per wavelength. However, modification of the element length does not have a strong effect on the accuracy of the integration; for more elements per wavelength the variation of the Hankel function across the interval is reduced. The error metric is taken as the percentage variation from a reference solution computed using adaptive numerical integration to a precision of $\sim 10^{-20}$. Fig. 2(a) shows how the error in the integration of Eq. (17) [using Eq. (5)] changes as the number of Gauss-points is increased for $\left(\xi_{i}, \mu_{i}\right)=(0,10)$. The convergence for $k=0.3$ ( $\sim 10$ elements per wavelength) is also shown for comparison. The error converges to machine precision very rapidly and the use of only 6 Gauss-points is sufficient to achieve a high level of integration accuracy.

\subsection{Computation of weakly singular integrals}

The primary numerical difficulty in the implementation of the direct collocation BEM is the evaluation of singular integrals. An integral is singular if at some point in the integration domain the integrand becomes infinite. Such integrals occur in the BEM when 
a

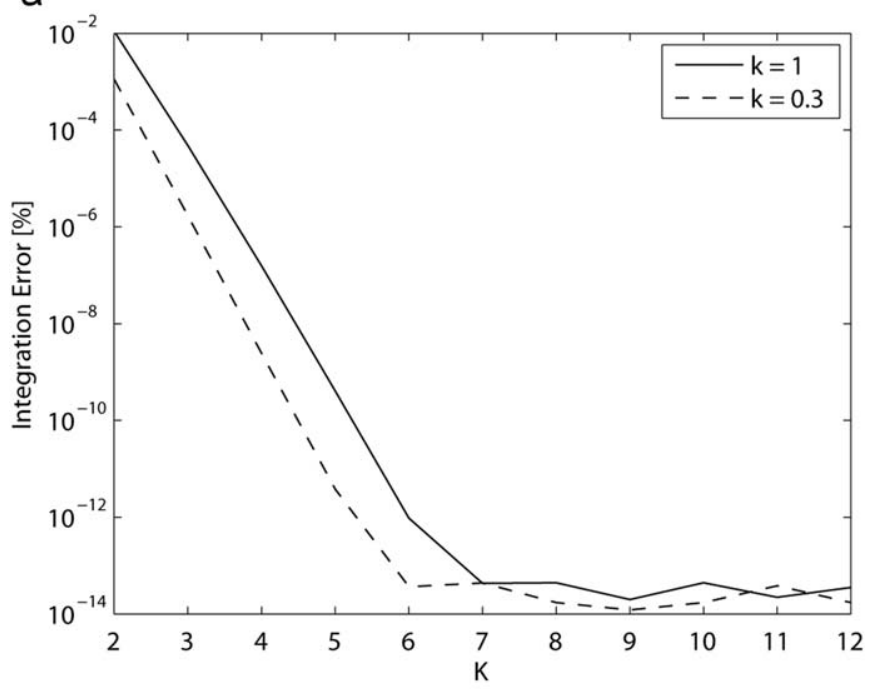

b

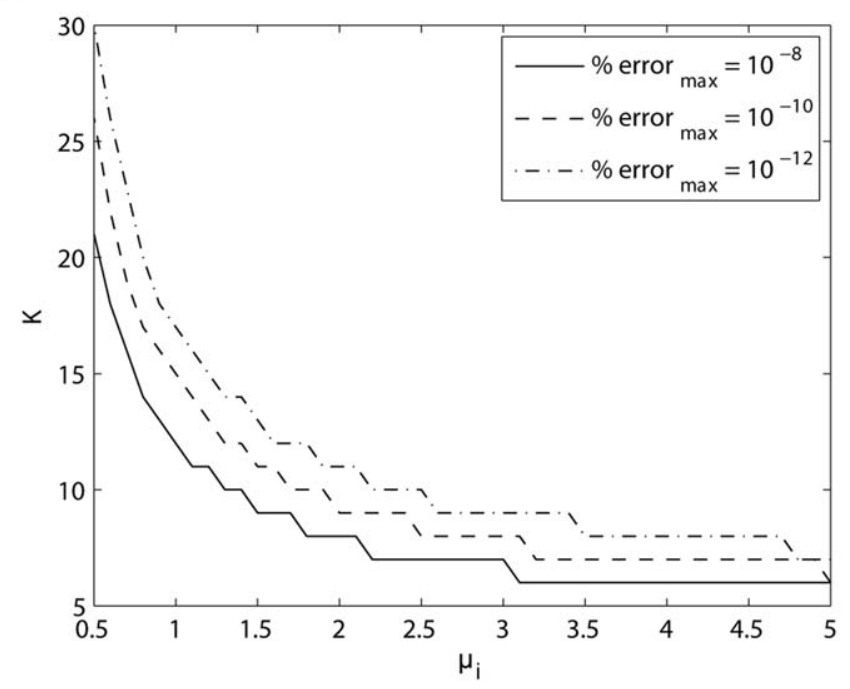

Fig. 2. (a) The error convergence of a regular integral with the total number of Gauss-points $K$ for two different wave-numbers, and (b) the number of Gausspoints $K$ required to maintain a given maximum error bound as the evaluation point $P_{i}=\left(0, \mu_{i}\right)$ gets closer to the integration element.

the evaluation point lies within the integration element, and thus the distance $r_{j i} \rightarrow 0$ within the Green's function and its derivatives [40]. Examining the integrals within the Helmholtz integral equation shown in Eq. (1) [or Eq. (7)], the integral of the Green's function derivative remains regular $[\partial G / \partial r$ is $O(1 / r)$, but $\partial r / \partial n$ is $O(r)]$. Conversely, the Green's function integral is weakly singular. Weakly singular integrals converge in the regular Riemann sense as the integral is continuous at the singular point (in contrast to strongly singular integrals which are unbounded at the singular point, i.e., the integral does not exist).

As the point $P_{i}$ approaches the integration element, the integral solution becomes dominated by the nearby singularity. This can be illustrated by again using the simplified boundary integral given in Eq. (17) and gradually moving $P_{i}$ closer to the element. To maintain a given level of accuracy, an asymptotically increasing number of Gauss-points are required. This is shown in Fig. 2(b), where nearly three times as many Gauss-points must be used when $P_{i}$ is within half an element length of the integration element. As $P_{i}$ continues to approach the element, the required increase in Gauss-points becomes excessive; if $P_{i}$ lies on the element, the integral becomes weakly singular and the regular
Gaussian quadrature is rendered ineffective for practical numbers of Gauss-points.

The numerical computation of singular integrals can be improved using a number of procedures. These include analytical evaluation [41,42], division of the interval about the singularity [43], subtraction of the singularity [42], special quadrature routines [44], and the use of non-linear coordinate transformations [45-49]. Each method has its own particular advantages and disadvantages depending on the type and order of the boundary elements used, and the form of the singular integral. The latter is also dependent on the partial differential equation being solved by the BEM. Here the analysis is restricted to non-linear coordinate transformations. Such transformations map the integration interval onto itself using a polynomial. They have the property of a null Jacobian at the singularity which weakens the singularity order. The transformations can be directly used with standard Gaussian quadrature routines, minimising the changes required to the BEM. Moreover, their application is not restricted to certain element or singularity types, making them extremely versatile.

Of the multitude of available non-linear coordinate transformations, that proposed by Sato and colleagues is amongst the most efficient [43]. The original Sato transformation of order $\sigma \geq 2$ is given by

$\xi(\gamma)=\bar{\xi}-\frac{\bar{\xi}}{2^{\sigma-1}}(1-\bar{\xi} \gamma)^{\sigma}$,

where $\gamma$ and $\xi$ represent the original and remapped Gauss-points, respectively, and the location of the singularity $\bar{\xi}$ must be \pm 1 (i.e., this particular transformation is only valid for endpoint singularities). For interior singularities, the interval must first be partitioned at the singularity, the transformation applied to both partitions, and the intervals then mapped back to [-1,1] [43]. For an arbitrary interior singularity located at $-1<\bar{\xi}<1$, this yields the following general transformation

$$
\begin{aligned}
I= & \frac{1+\bar{\xi}}{2} \int_{-1}^{1} f\left(\frac{(1+\bar{\xi})\left(1-(1-\gamma)^{\sigma} / 2^{\sigma-1}\right)+\bar{\xi}-1}{2}\right) \frac{\sigma(1-\gamma)^{\sigma-1}}{2^{\sigma-1}} d \gamma \\
& +\frac{1-\bar{\xi}}{2} \int_{-1}^{1} f\left(\frac{(1-\bar{\xi})\left((1+\gamma)^{\sigma} / 2^{\sigma-1}-1\right)+\bar{\xi}+1}{2}\right) \\
& \times \frac{\sigma(1+\gamma)^{\sigma-1}}{2^{\sigma-1}} d \gamma .
\end{aligned}
$$

Even for algorithms suitable for interior point singularities, partitioning can often improve the accuracy of the solution for a given number of overall Gaussian operations [43]. Note, that for an endpoint singularity, the Sato transformations for $\sigma=2,3$ are identical to the popular transformations proposed by Telles [45].

The efficiency of the Sato transformation for weakly singular integrals with a logarithmic singularity and real-valued kernels has been investigated previously [43]. However, the application of coordinate transformations for complex valued kernels does not always yield the expected results. For example, Elliott and Johnston comment that their sinh transformation must only be applied to the imaginary component of the Hankel function [50]. The efficiency of the Sato transformation is examined here by computing Eq. (17) when $P_{i}$ lies on the integration element (i.e., $\mu_{i}=0$ and $\left.0 \leq \xi_{i} \leq 1\right)$. Fig. 3(a) illustrates the error in the integral solution against the location of the singularity $\bar{\xi}$. As expected, the regular Gaussian quadrature is unable to compute the integral accurately, even using 20 Gauss-points. The application of the 3rd-order Telles transformation $[45,47]$ for the same number of Gauss-points reduces the integration error by several orders of magnitude. If the integral is partitioned at the singularity and Sato 

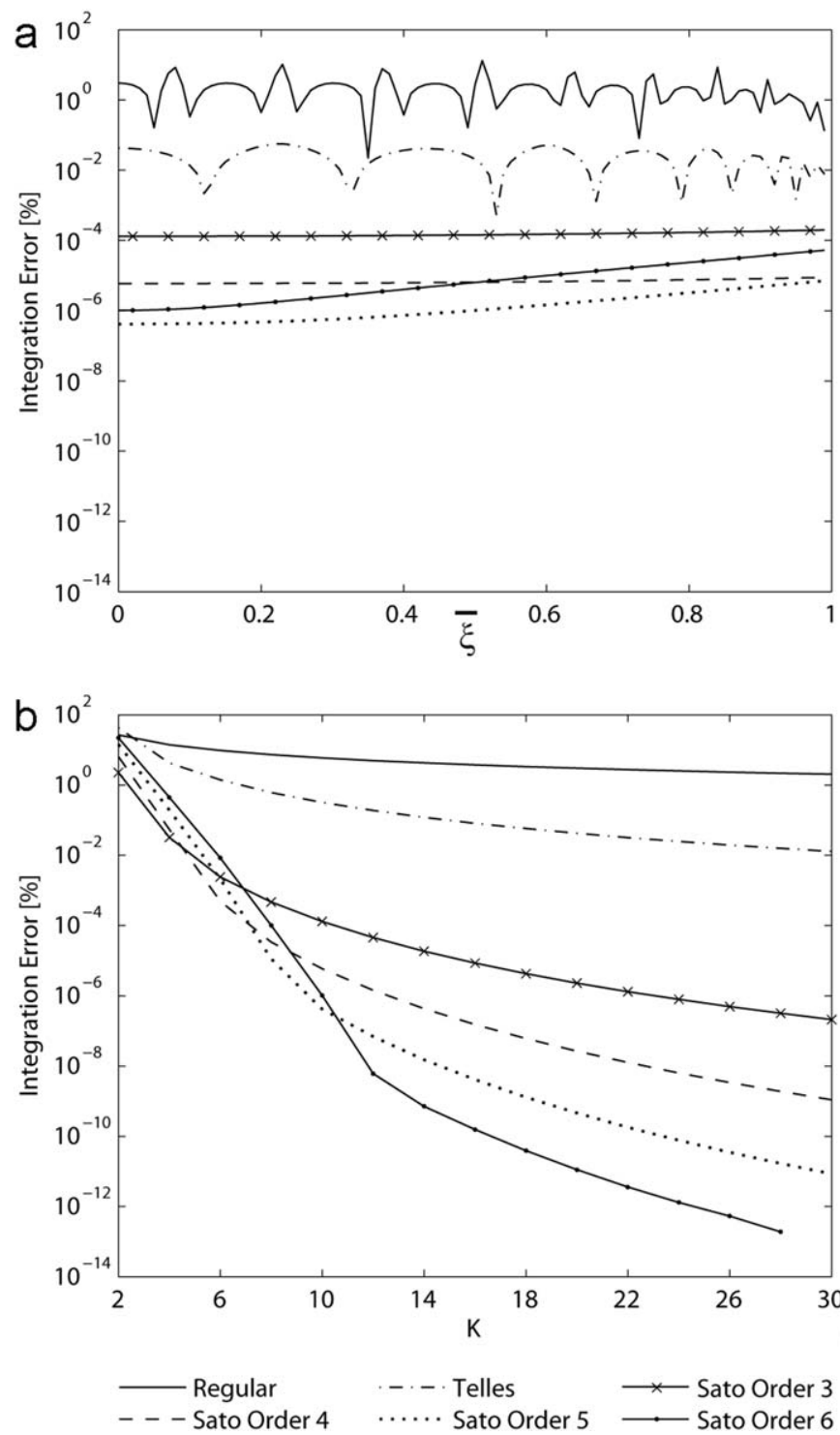

Fig. 3. Effect of non-linear coordinate transformations on the error in the computation of a weakly singular integral: (a) error variation with the location of the singular point $\bar{\xi}$ using a total of 20 Gauss-points, and (b) error convergence for an origin singularity with the number of Gauss-points $K$ per partition.

transformations of increasingly higher order are applied (still using a total of 20 Gauss-points, 10 per partition), the error is decreased further. This illustrates the usefulness of the transformation.

The convergence of the Sato transformation with the number of Gauss-points in each partition for various transformation orders is illustrated in Fig. 3(b). The transformations first display exponential followed by algebraic convergence. For small numbers of Gauss-points, the lowest level of error is not necessarily given by the transformation with the highest order; it is preferable to utilise the transformations within the second region of convergence. This is also evident in Fig. 3(a), where for 10 Gauss-points per partition, the 6th-order transformation produces a higher level of error than the 5th-order transformation. As higher-order transformations strongly cluster the Gauss-points around the singularity, if only a small number of Gauss-points are used this does not leave sufficient points distributed across the remainder of the integration interval to compute it accurately. A similar situation occurs for singularities near the element endpoints; if the number of Gauss-points used in each partition are divided evenly, the integration over the longer interval may not be computed with comparable accuracy. This is visible in Fig. 3(a) where the higher-order Sato transformations display a positive gradient as $\bar{\xi} \rightarrow 1$. Care must also be taken to avoid large numbers of Gauss-points with high transformation orders, as this can produce unstable behaviour [43] (see also discussion in [51]). For the purpose of dissociating integration and discretisation errors, a 5th-order Sato transformation coupled with 20 Gauss-points in each partition is sufficient to ensure the accurate computation of weakly singular integrals (while avoiding the instabilities of the transformation).

The singularity within the Hankel function kernel arises because of the origin singularity of the Bessel function of the second kind. Therefore, the coordinate transformation only needs to apply to the singular part of the Hankel function. However, for higher numbers of Gauss-points [the second region of convergence shown in Fig. 3(b)], there is no difference in the integration error if the integrals are computed using separated quadrature routines (regular and singular, respectively) for Bessel functions of the first and second kind. This is not true when using small numbers of Gauss-points. The extreme clustering of the integration points about the singularity means that the regular part of the integral is computed less accurately than if the points had been distributed evenly. In this case, the transformation should only be applied to the singular component of the integral. A similar effect is described by Elliot and Johnson in relation to their $\sinh$ transformations used for nearly weakly singular integrals [50].

Finally, it is important to note that the primary motivation behind using non-linear coordinate transformations is that the Gaussian quadrature routines (including the number of Gausspoints) used for regular integrals can also be utilised for singular integrals with no modification [52]. The question of whether singular integrals can be computed with sufficient accuracy using 6 Gauss-points (which evidently is adequate to compute regular integrals accurately) will be discussed in Section 6. As the remaining integral term within Eq. (7) is regular, the location of the evaluation point $P_{i}$ in relation to the element does not affect the convergence of the quadrature routine and thus no modification is required.

\subsection{Computation of nearly singular integrals}

While the non-linear coordinate transformations just discussed are able to compute weakly singular integrals successfully, when the evaluation point $P_{i}$ is near (but not on) the integration element these transformations do not necessarily provide the same advantages [53]. For nearly weakly singular integrals, the transformations must instead contain a modifiable Jacobian that is close to (but not exactly) zero at the nearly singular point. The strength of the quadrature point clustering can then be related to the strength of the near-singularity via the distance between the evaluation point and the integration element. (Note, although element subdivision is also commonly utilised for dealing with nearly singular integrals, these routines are not computationally economical and additionally restrict the order of the function that can be exactly computed for a given number of total Gausspoints.).

The most widely used non-linear coordinate transformation for nearly weakly singular integrals was proposed by Telles $[45,47]$ This utilises an optimisation parameter $\bar{r}$ that is dependent on both the order of the singularity and the closest distance $D$ from the element to the near singular point [45,47]. Using the optimised $\bar{r}$ values given in Ref. [47], an appropriate expression for $\bar{r}$ dependent on the distance parameter $D$ is given by 
$\bar{r}=e^{\wedge}\left(-2.2 D^{2}-4.8 D-0.34\right)^{-1}$. This may be used in conjunction with Telles adaptive transform to adjust the optimisation parameter automatically. However, previous investigations have illustrated the strong sensitivity of the integration accuracy to the optimisation parameter [23], and recently other transformations suitable for computing nearly weakly singular integrals have also been proposed [54,55]. In particular, the sinh transformation by Johnston and Elliot provides a useful alternative $[50,55,56]$.

The effect of these transformations on the accuracy of nearly singular integrals can again be examined using the integral simplification given in Eq. (17). Fig. 4(a) illustrates how the error changes when the evaluation point approaches the centre of the element [i.e., $\left.\left(\xi_{i}, \mu_{i}\right)=(0, D)\right]$ and Fig. $4(\mathrm{~b})$ the right-hand side [i.e., $\left.\left(\xi_{i}, \mu_{i}\right)=(1+D, 0)\right]$. Both the $\sin h$ and Telles transformations provide a significant improvement in integration accuracy compared to the regular quadrature (each using a total of 20 Gauss-points, except for the $K=6$ reference). It is also clear that if the nearly singular point is located towards the element endpoints, the effect on the integration accuracy is reduced. In both cases, the sinh transformation provides a reasonable improvement on Telles' transformation.

The convergence of these transformations with the number of Gauss-points is provided in Fig. 4(c) (Telles), and Fig. 4(d) (sinh).
Again, for low numbers of Gauss-points, it is recommended to only apply the transformation to the singular part of the Hankel function integral kernel. Similarly, for higher numbers, this has a negligible effect on accuracy. As the parameters needed for the $\sinh$ transformation may be pre-computed using the boundary geometry, it is legitimate to always use the transformation within the computation of the second integral term of Eq. (7). This does not significantly affect the computational time, as any use of the sinh transformation already requires the computation of the element-wise arrays of $D$ and $\bar{\xi}$. Again, for the purpose of dissociating integration and discretisation errors a $\sin h$ transformation with 20 Gauss-points is sufficient to accurately compute the encountered nearly weakly singular integrals.

Before beginning the next part of the analysis, it is useful to note that other numerical procedures could alternatively be used to compute the required element-wise integrations. In the context of the wider investigation, this is of no concern, as it is also the absolute level of accuracy required by these routines that is also under consideration. Gaussian quadrature and non-linear coordinate transformations simply provide an efficient and effective way of computing these integrals. Moreover, by modifying the total number of Gauss-points they also provide a systematic way for the accuracy (and correspondingly, the computational expense) to be altered.

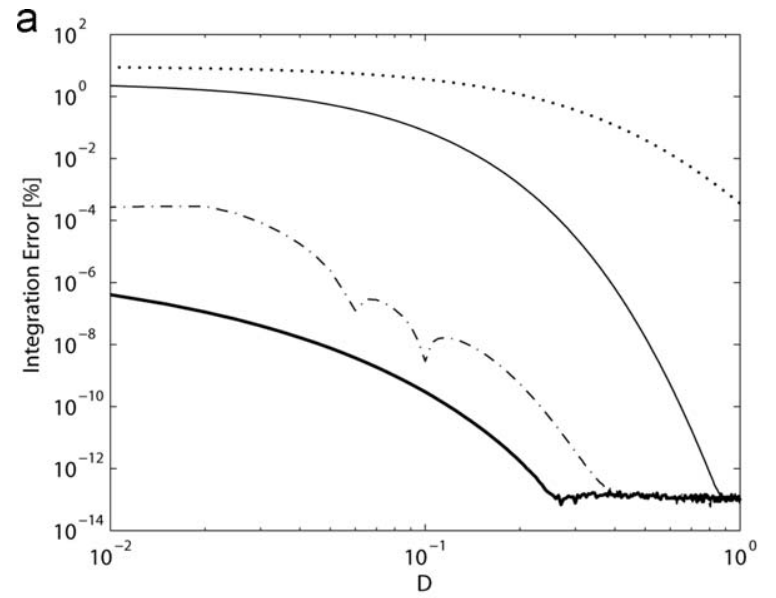

b
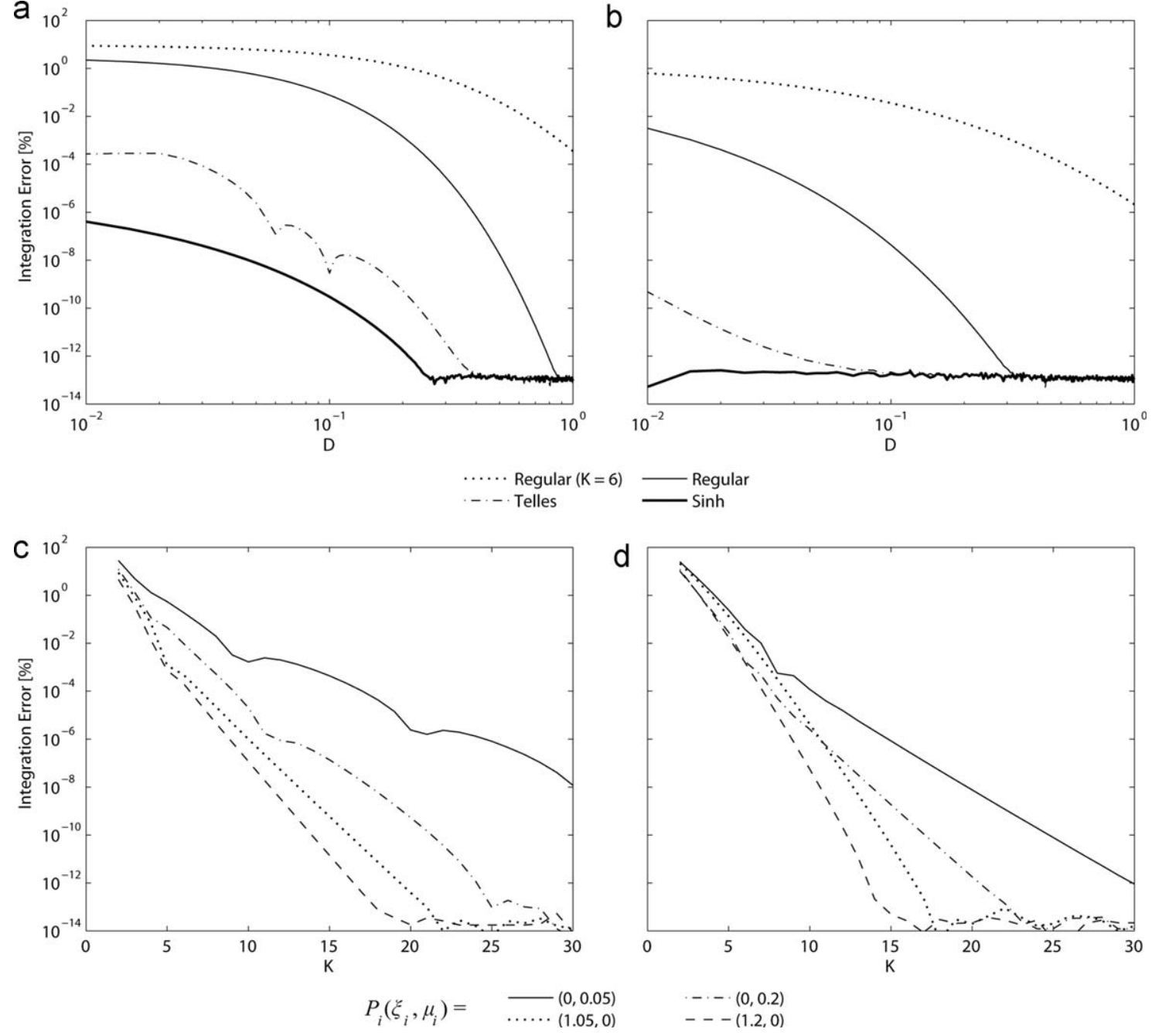

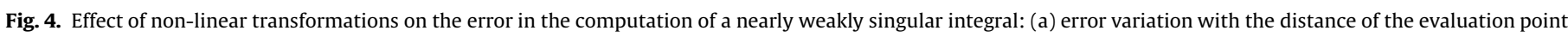

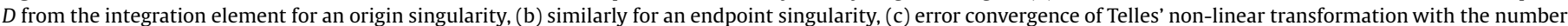
of Gauss-points $K$ for various locations of the evaluation point, and (d) similarly for the sin $h$ transformation. 


\section{The effect of discretisation error}

Now that numerical routines for computing element-wise integration have been discussed, it is appropriate to investigate the effect of element type and boundary discretisation on the global error of an objective problem. A suitable 2D reference is the scattering of an infinite line source by a parallel infinite cylinder. Using the expansion of a uniform outgoing cylindrical wave (via Fourier transformation) by Morse and Ingard [57], an analytical expression for the total pressure surrounding the scatterer can be derived. For a passive Robin boundary condition this is given by

$$
\begin{aligned}
p= & \sum_{n=0}^{\infty} P_{i} \varepsilon_{n}\left[J_{n}\left(k r_{\text {inf }}\right)-\frac{J_{n}{ }^{\prime}(k a)+i \beta J_{n}(k a)}{H_{n^{\prime}}(k a)+i \beta H_{n}(k a)} H_{n}\left(k r_{\text {inf }}\right)\right] \\
& \times H_{n}\left(k r_{\text {sup }}\right) \cos (n \varphi),
\end{aligned}
$$

where $r_{\text {inf }}$ is the infimum of $\left(r, r_{0}\right)$ (i.e., the smaller of $r$ and $\left.r_{0}\right), r_{\text {sup }}$ the supremum of $\left(r, r_{0}\right)$ (i.e., the larger of $r$ and $\left.r_{0}\right), \varepsilon$ the Neumann factor given by $\varepsilon_{n=0}=1$ and $\varepsilon_{n \geq 1}=2, J_{n}$ the Bessel function of the first kind, the ' operator represents the radial derivative, and the other parameters are defined in Fig. 5 and previous sections (similar analytical reference problems also exist in 3D [58]).

To examine the convergence of the BEM for various element orders, the direct collocation BEM described in Section 2 is used to numerically compute the domain pressure at a series of near-field evaluation points. These reference points are positioned evenly on concentric circles of radii $2 a, 3 a$, and $4 a$, with 32 points on each. The error metric is taken as the percentage error relative to the analytical solution [given by Eq. (20)] averaged across these locations. While this choice of error metric is somewhat arbitrary, the utilised reference points are representative of the data often required from acoustic scattering analyses. The use of other metrics may give slightly varying absolute error values, however, the observed trends remain robust. For the present investigation, it is sufficient to use a Neumann boundary condition with $\beta=0$ (i.e., a rigid cylinder) as this removes the contribution of the singular integral from Eq. (7). Similarly, the use of parabolic continuous shape functions to model the boundary geometry dissociates errors in the discretisation of the curved boundary.

The global error convergence with the number of nodes per wavelength (rather than elements per wavelength) for constant, linear, and parabolic variations of the boundary quantities is shown in Fig. 6(a). This allows a legitimate comparison of the accuracy across differing element orders. A non-dimensional wave-number of $k a=16$ (with $r_{0}=20$ ) is used to allow a smooth variation of the corresponding number of elements required. As expected, the refinement method exhibits an algebraic convergence that is related to the element order [8].

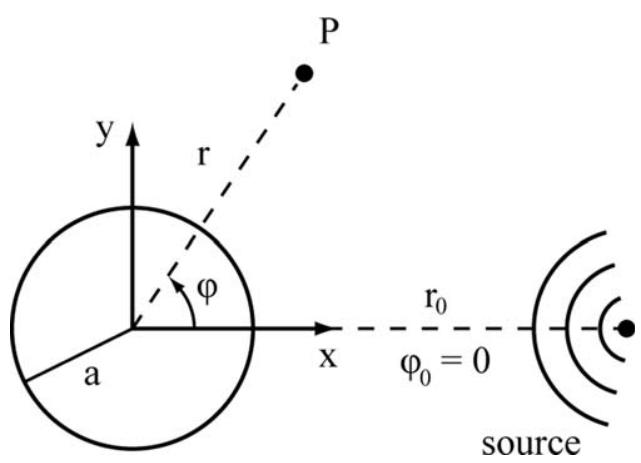

Fig. 5. Infinite cylinder reference problem. The source is located on the positive $x$-axis and the evaluation position is denoted by $P$. There are $3 \times 32$ evaluation positions used to compute the error metric spaced evenly on concentric circles with radii of $2 a, 3 a, 4 a$.

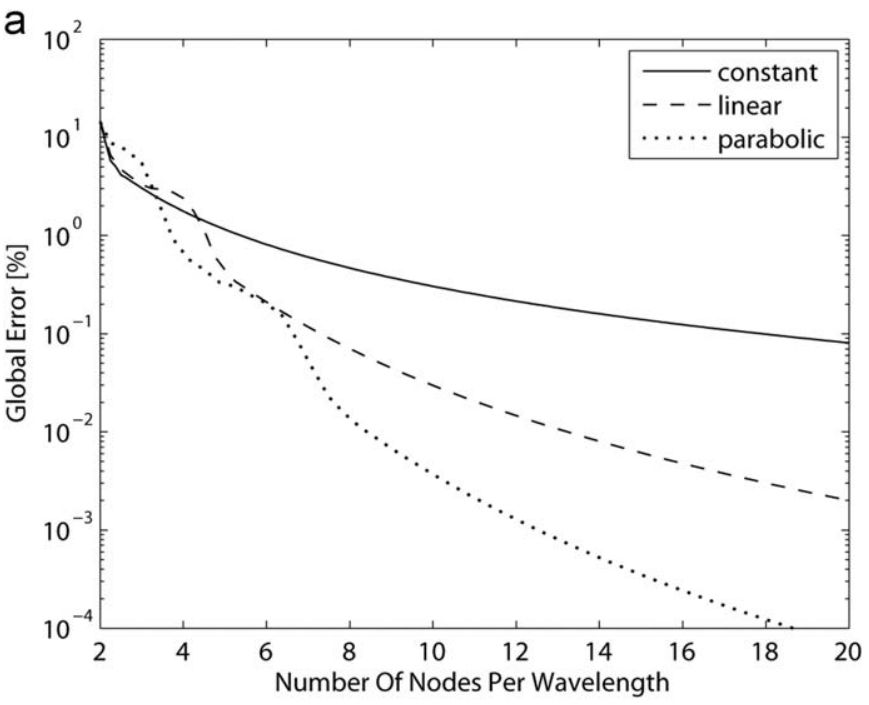

b

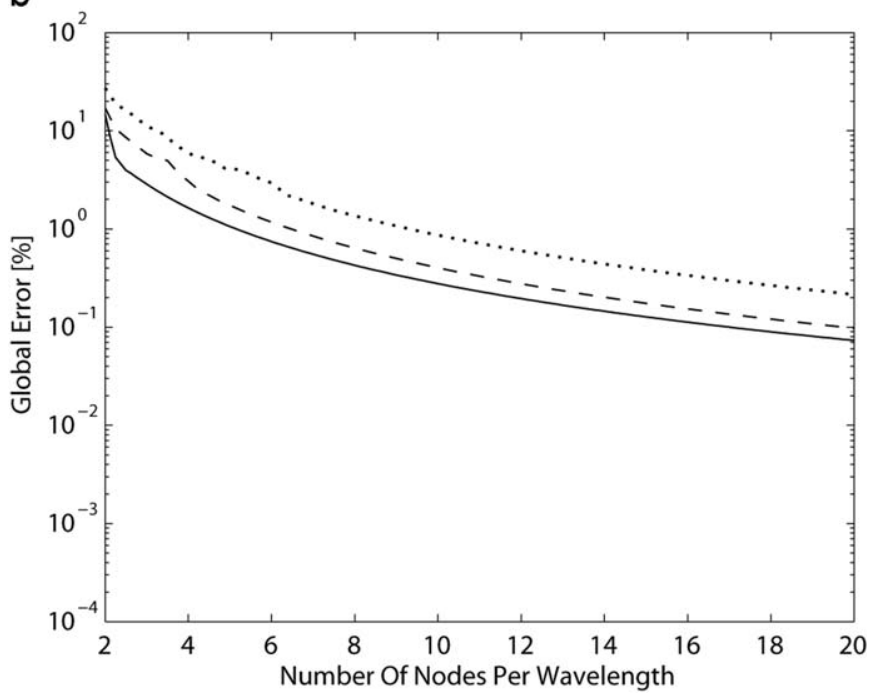

Fig. 6. Global error convergence with the number of nodes per wavelength for constant, linear, and parabolic elements for a cylindrical scattering problem: (a) using parabolic shape functions for the geometric discretization, and (b) similarly using linear shape functions.

For this smoothly varying geometry, the application of 6 constant elements per wavelength produces a global error metric in the region of $1 \%$. This corresponds to the recommendation commonly given for engineering applications. For the same computational expense, the use of parabolic elements reduces this error to approximately $0.2 \%$; to yield $1 \%$ error requires only 4 nodes per wavelength.

For comparison, Fig. 6(b) shows the analogous results using linear continuous shape functions to describe the variation of the boundary geometry. There is no longer an advantage in increasing the number of nodes per element. In this case, the error in the geometric discretisation becomes the limiting factor in the convergence. For curved objects, it is therefore strongly recommended that parabolic shape functions are used to model the boundary geometry.

\section{The effect of geometric discontinuities}

The cylindrical scatterer examined in the previous section has a very regular geometry, and consequently, a smoothly varying surface pressure. While this allows a useful comparison to an 
analytical solution, it is not particularly representative of objective BEM problems. When the boundary contains abrupt changes or discontinuities in the profile or surface properties, the resulting surface pressure can vary rapidly. In the case of discontinuous elements, the continuity of the surface pressure across these singularities is identifiably not enforced. This can create large discontinuous jumps in the boundary quantities which in turn reduces the accuracy of the boundary integration. To illustrate the effect of boundary singularities on convergence and accuracy, it is

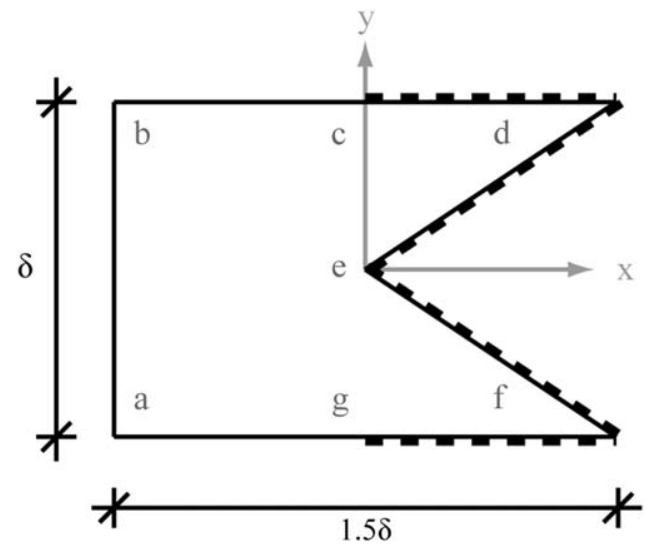

Fig. 7. Irregular scatterer with discontinuities in both the boundary shape and surface properties. The solid lines represent rigid boundaries, and the dashed lines absorbing boundaries with an admittance of $\beta=0.5-0.5 i$. useful to examine a more complex scattering object as shown in Fig. 7. This is a rectangle of dimensions $\delta \times 1.5 \delta$ with a triangular cavity and a discontinuous surface admittance (denoted by the broken lines). The boundary changes are denoted $a, b, \ldots, g$ and the source is taken to be located at $(x, y)=(20 \delta, 0)$.

Fig. 8 shows the surface pressure for $k d=2$ using parabolic [Fig. 8(b)], linear [Fig. 8(c)], and constant [Fig. 8(d)] boundary elements with approximately the same number of total nodes. Continuous linear shape functions are used to model the geometric variation. The converged surface pressure is shown in Fig. 8(a). It is clear, at least visually, that higher-order elements approximate the surface pressure more closely. Fig. 9(a) illustrates the global error convergence for the three examined element orders with the number of nodes per wavelength. The same error metric as in Section 4 is used, but with the reference points positioned on concentric circles of radii $1.5 \delta, 2.5 \delta$, and $3.5 \delta$. Increasing the element order still provides an increase in accuracy, but the boundary discontinuities eliminate the convergence advantages. For parabolic elements, 4 nodes per wavelength remains adequate to maintain a global error level around $1 \%$.

If a higher level of accuracy is required, simple a priori mesh grading techniques are often sufficient to improve the global error convergence (and thus computational efficiency) with the number of nodes per wavelength. Instead of dividing the boundary sides into $l=0,1, \ldots, L$ uniformly spaced divisions (i.e., for a side defined by $0 \leq \eta \leq 1$, the divisions are given by $\eta_{l}=l / L$ ), the elements are graded towards an endpoint singularity based on a grading parameter $q \geq 1$, where $\eta_{l}=\left|b-(b-l / L)^{q}\right|$, and $b=0$ or 1 for singularities located at $\eta=0$ or 1 , respectively.

b

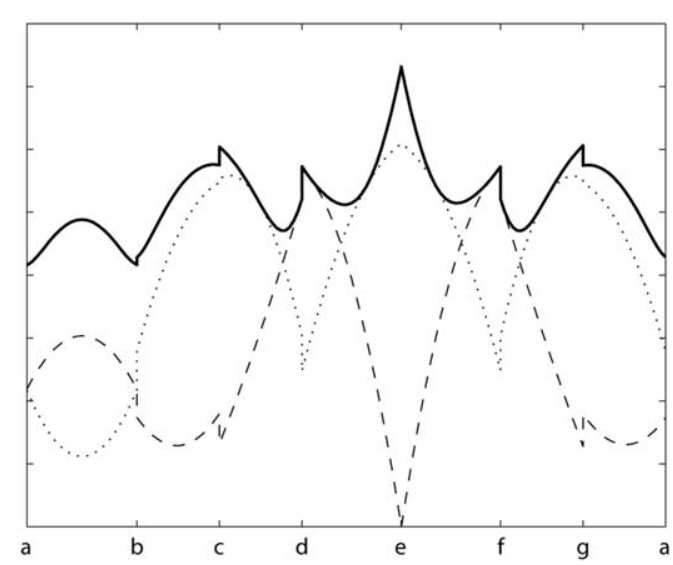

d

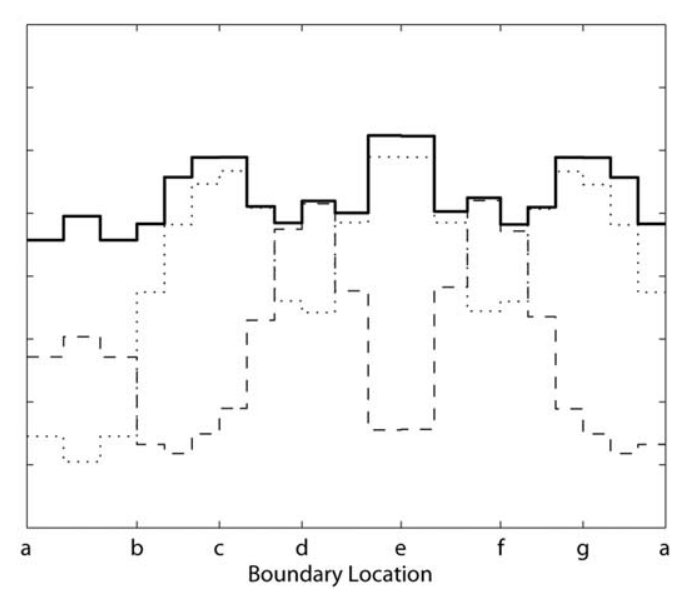

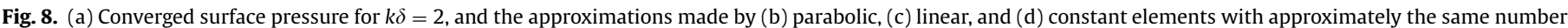
of total nodes. 

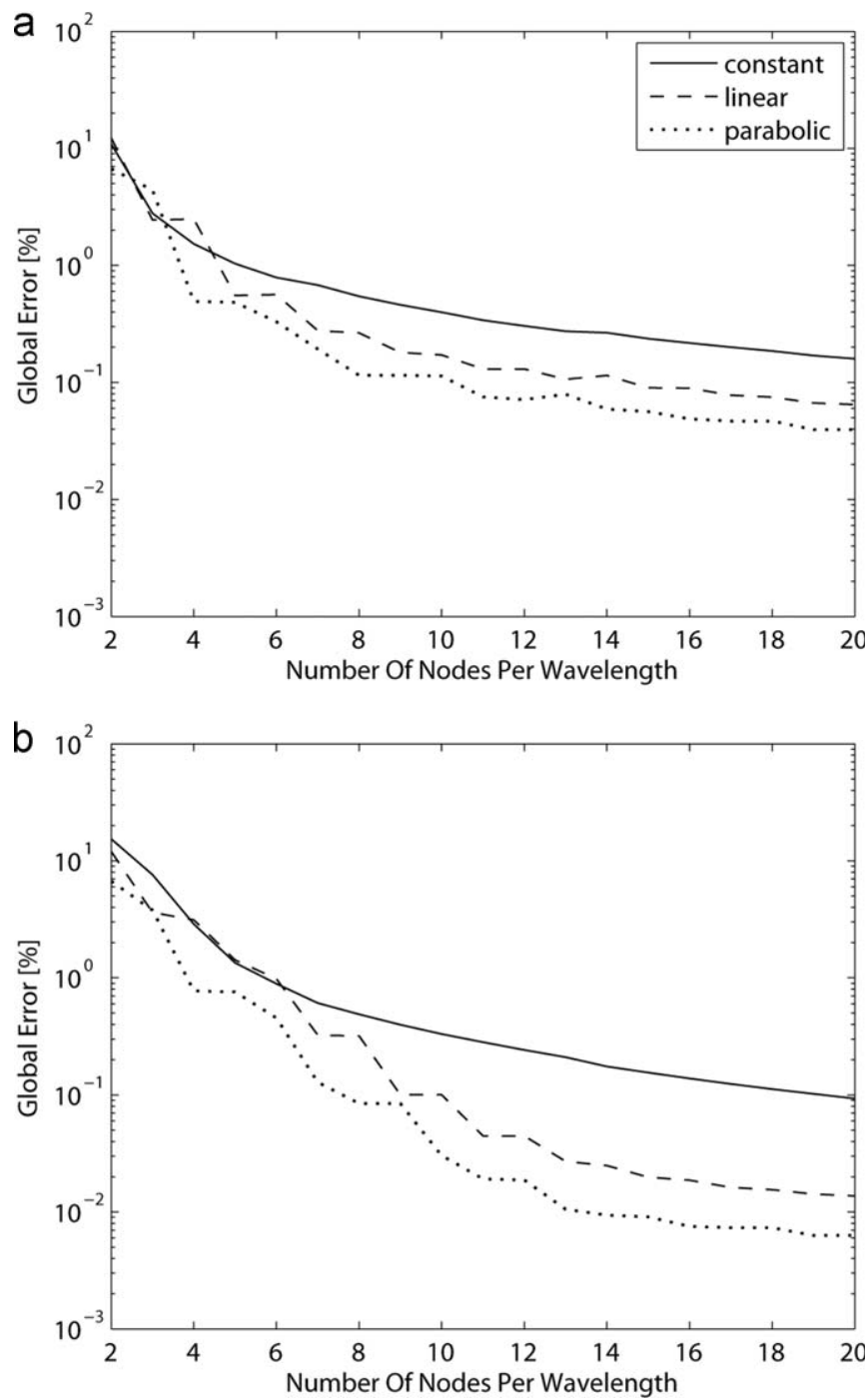

Fig. 9. Error convergence with the number of nodes per wavelength for constant, linear, and parabolic elements for an irregular scatterer; (a) using an evenly divided mesh, and (b) using an a priori mesh grading with $q=2$.

For the example used here, each boundary length (i.e., $\overrightarrow{a b}, \overrightarrow{b c}$, etc.; see Fig. 7) has boundary disparities at both endpoints. The lengths are thus first divided into two, and the elements evenly graded towards the discontinuities. As this is only a variation to the input mesh, no modification to the BEM is required. The modified error convergence for $q=2$ is shown in Fig. 9(b). For higher numbers of nodes per wavelength, the grading has restored the convergence of linear elements and improved that of the parabolic. Note, if high levels of accuracy are required for particularly discontinuous boundaries, adaptive mesh refinement procedures can provide a more notable enhancement to the solution convergence. Such an approach removes the need to scale meshes arbitrarily without a priori information about the surface quantity variation. However, the corresponding increase in computational effort may be disproportionate considering the small number of nodes per wavelength typically required for engineering-type accuracies.

\section{The effect of integration error}

In Section 3, high-order quadrature routines were established to compute the required element-wise integration tasks accurately.
In the subsequent sections, these were utilised to dissociate integration errors from the discretisation errors under examination. It is now important to establish the actual integration accuracy that is required for a given global error constraint. The appropriate quadrature complexity can then be selected accordingly, minimising any unnecessary computational expense.

Fig. 10 illustrates the relationship between the global error and the integration accuracy for the irregular scatterer (and error metric) discussed in Section 5 [Fig. 10(a) and (b)], and the cylindrical scatterer discussed in Section 4 [Fig. 10(c) and (d)]. Parabolic shape functions are used to describe the boundary quantities, with linear (irregular) and parabolic (cylinder) shape functions to describe the geometric variation. The integration accuracy is adjusted by polluting the result of each quadrature computation with a random number (scaled to a particular maximum). Fig. 10(a) and (b) correspond to independent adjustment of integration accuracy for regular and weakly singular integrals, respectively, with the alternate parameter not being modified. The remaining plots correspond to the adjustment of integration accuracy for regular integrals only, using an absorbent [Fig. 10(c)] and a rigid [Fig. 10(d)] cylinder. For each plot, 10 curves are displayed using 3 to 12 nodes per wavelength (higher numbers of nodes per element correspond to lower curves).

It is immediately evident that the global error metric has a stronger dependence on regular integration accuracy than weakly singular integration accuracy [Fig. 10(a) and (b)]. The exact dependence on the latter is governed by the dominance of the diagonal terms in the coefficient matrix $\mathbf{A}$. This is reliant on the shape of the scatterer and the imposed surface boundary conditions. However, the explanation for the preferential dependence seen here is more straightforward. Weakly singular integrals are computed during the initial calculation of the boundary quantities (via collocation) but not during the calculation of the pressure within the domain. Regular integrals are involved in the computation of both. As the utilised error metric consists of points within the scattering domain, the pollution of the regular integration computations has a larger effect.

For the cylindrical scatterers [Fig. 10(c) and (d)], the error dependence is analogous, although, a more rapid error convergence is evident with the number of nodes per wavelength, and the absolute dependence on integration accuracy is slightly increased. This is due to the smooth cylindrical geometry (there are no boundary discontinuities). An increase in global error with an increase in the number of nodes per wavelength can also be seen for very low levels of integration accuracy. Overall, the absolute integration accuracies required to maintain a given global error bound are reasonably low. This requirement is systematically increased when the number of nodes per wavelength or the element order are increased.

For the scattering examples investigated here, completely removing compensation for nearly weakly singular integrals has no observable effect on accuracy. Evidently, the decrease in integration accuracy for the small number of nearly weakly singular kernels is not sufficient to affect the global error values. In the context of the discussion given in Section 3, the strength of the near-singularity for adjacent elements of identical size is low. Moreover, there are only a small number of vertices in relation to the number of elements distributed on planar surfaces. Consequently, for the types of boundary meshes considered, the inclusion of distinct routines for the computation of nearly weakly singular integrals is not required. Although, for boundary meshes with large element length disparities, this may no longer be true (the distance to the nearest node in relation to the element length may become very small). 


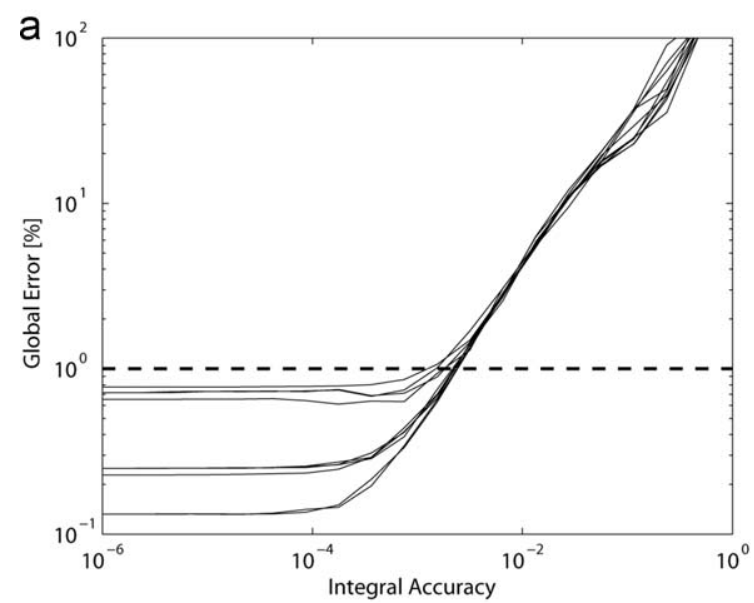

b
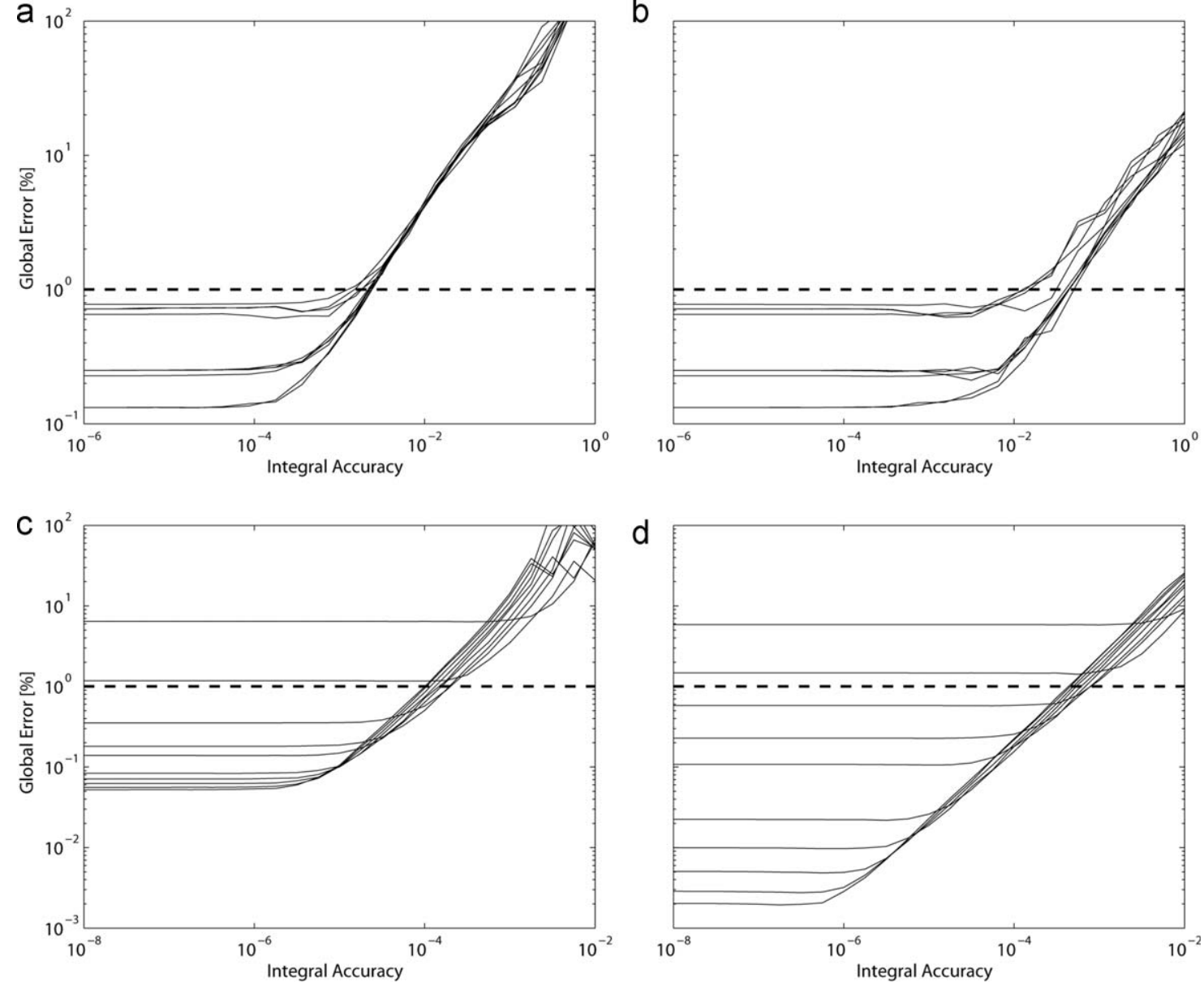

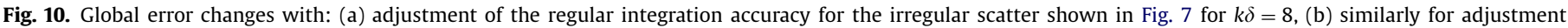

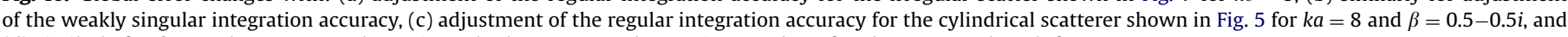
(d) similarly for $\beta=0$. The 10 curves shown in each plot correspond a varying number of nodes per wavelength from 3 to 12 .

At this point it is constructive to collect the results of the current and previous sections into a set of empirical BEM guidelines. For a global error requirement of $\sim 1 \%$, the appropriate discretisation and computational parameters are:

- a uniform boundary mesh with 4-6 nodes per wavelength (using discontinuous parabolic elements; see Figs. 6 and 9);

- and, 6 point Gaussian quadrature for the computation of regular integrals, with the application of a 4th-order Sato transformation for weakly singular integrals [see Figs. 10, 2 and 3]. No treatment is required for nearly weakly singular integrals.

To illustrate the efficacy of these guidelines, a final scattering example is taken as shown in Fig. 11(a). The change in domain pressure at two arbitrary locations with the non-dimensional wave-number $k \delta$ (using the parameters above with 4 nodes per wavelength) is shown in Fig. 11(b). The converged numerical result is shown for comparison. For these particular domain points, the use of 4 nodes per wavelength restricts the error to $\sim 2-5 \%$; for 6 nodes per wavelength this is reduced to $\sim 1-3 \%$. Using the figures and analyses provided here, it is straightforward to derive similar guidelines for alternate global error requirements.

\section{Summary and discussion}

The continued interest in the BEM as a computational analysis tool over the last decade has predictably yielded a large number of academic investigations into the methodology. New algorithms and techniques have been proposed to extend the functionality of the BEM and compute the numerical tasks with ever increasing accuracy. On the surface, these developments appear intrinsically useful. Indeed, the overall error in a BEM simulation is dependent on both discretisation and computational errors, in addition to the non-uniqueness problem. However, the exact accuracy requirements for each of the computational constituents is frequently overlooked.

In this context, the discretisation and computational errors for the direct collocation BEM are explored here in a necessarily parallel fashion. First, methods to compute the required numerical integration tasks using Gaussian quadrature and non-linear coordinate transformations are discussed. In particular, the accuracy and convergence of these routines for regular, weakly singular, and nearly weakly singular kernels is established. Subsequently, the effect of the boundary discretisation on global error values is illustrated. Finally, the actual integration accuracy required to maintain a given global error is considered. In the context of the continued expansion of computational literature, it is the latter that is most pertinent. For the BEM formulation and 


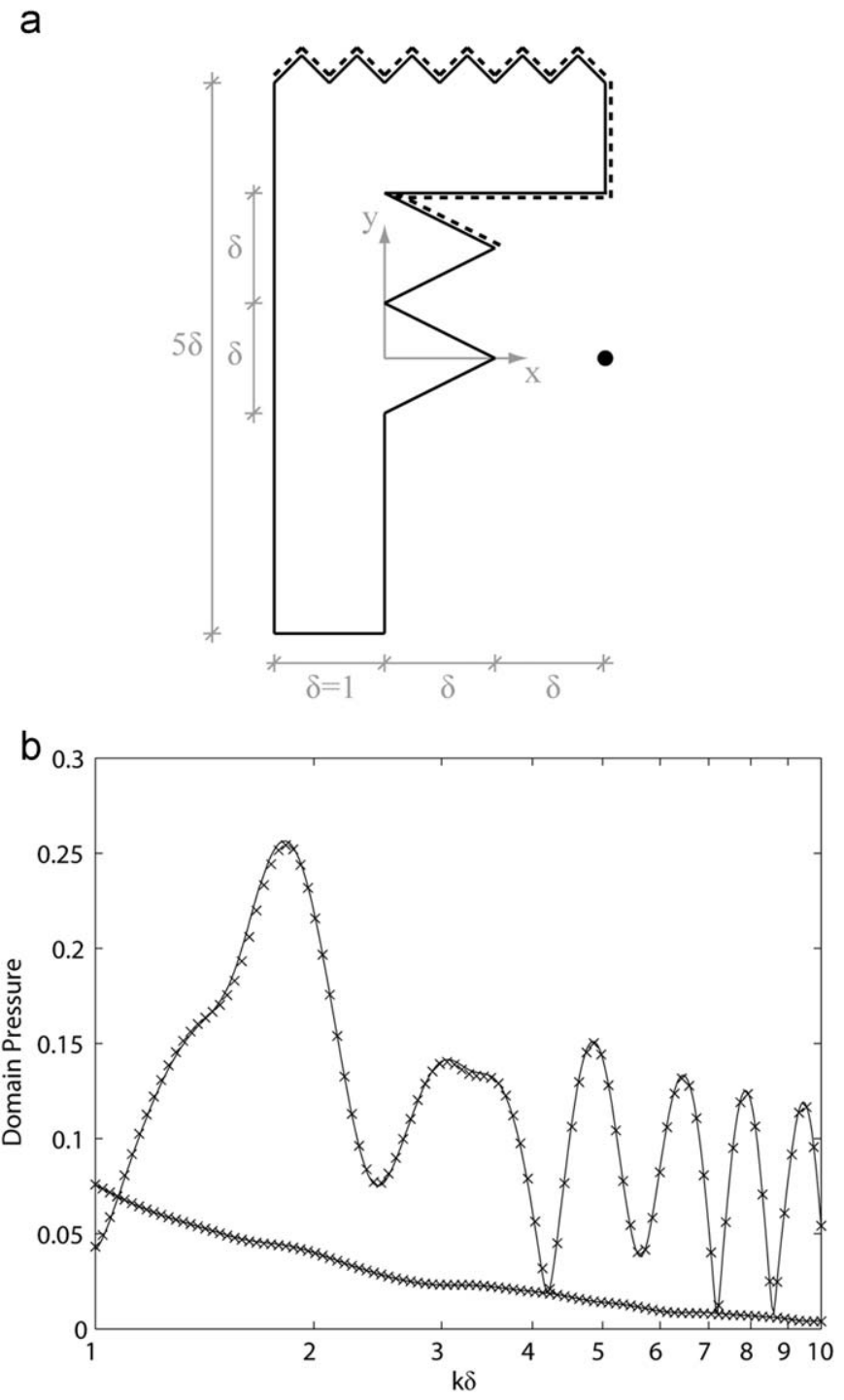

Fig. 11. (a) Irregular scattering example with discontinuities in both the boundary shape and surface properties. The solid lines represent rigid boundaries, and the dashed lines absorbing boundaries with an admittance of $\beta=0.5-0.5 i$. (b) Domain pressure at $(x, y)=(2,0)$ (upper curve) and $(-20,0)$ (lower curve) for a point source positioned at $(20,0)$ assuming $\delta=1$. The crosses indicate the numerical results using the parameter guidelines and the solid line the converged numerical result.

acoustical scatterers examined here, the integration accuracy requirements can be met with modest computational force. That is, for engineering-type accuracies, the global error is overwhelmingly dictated by the fineness of the boundary discretisation. Using the provided analyses, it is straightforward to extract empirical parameter guidelines for a given global error requirement. This is particularly important for optimising the efficiency of the BEM while maintaining a desired accuracy.

\section{References}

[1] Arnold D, Wendland W. On the asymptotic convergence of collocation methods. Math Comput 1983;41(164):349-81.

[2] Wendland WL. Boundary element methods and their asymptotic convergence. In: Filippi P, editor. Theoretical acoustics and numerical techniques. New York: Springer; 1983. p. 135-16.

[3] Marburg S. Six boundary elements per wavelength: is that enough?. J Comput Acoust 2002;10(1):25-51.

[4] Ciskowski RD, Brebbia CA. Boundary element methods in acoustics. Southampton: Computational Mechanics Publications; 1991.

[5] Chen G, Zhou J. Boundary element methods. London: Academic Press; 1992.
[6] Wu TW, editor. Boundary element acoustics: fundamentals and computer codes. Ashurst: WIT Press; 2000.

[7] Katsikadelis JT. Boundary elements: theory and applications. Oxford: Elsevier; 2002.

[8] Juhl P. A note on the convergence of the direct collocation boundary element method. J Sound Vibr 1998;212(4):703-19.

[9] Marin L, Lesnic D, Mantic V. Treatment of singularities in Helmholtz-type equations using the boundary element method. J Sound Vibr 2004;278(1-2): 39-62.

[10] Rank E. Adaptive h-, p- and hp-versions for boundary integral element methods. Int J Numer Methods Eng 1989;28:1335-49.

[11] Holm H, Maischak M, Stephan E. The hp-version of the boundary element method for Helmholtz screen problems. Computing 1996;57(2):105-34.

[12] Marburg S, Schneider S. Influence of element types on numeric error for acoustic boundary elements. J Comput Acoust 2003;11(3):363-86.

[13] Liapis S. A review of error estimation and adaptivity in the boundary element method. Eng Anal Boundary Elem 1994;14(4):315-23.

[14] Guiggiani M. Error indicators for adaptive mesh refinement in the boundary element method-a new approach. Int J Numer Methods Eng 1990;29(6): 1247-1269.

[15] Liang M, Chen J, Yang S. Error estimation for boundary element method. Eng Anal Boundary Elem 1999;23(3):257-65.

[16] Chen JT, Chen KH, Chen CT. Adaptive boundary element method of timeharmonic exterior acoustics in two dimensions. Comput Methods Appl Mech Eng 2002;191(31):3331-45.

[17] Kita E, Kamiya N. Error estimation and adaptive mesh refinement in boundary element method, an overview. Eng Anal Boundary Elem 2001;25(7):479-95.

[18] Ke C, Amini S. Numerical analysis of boundary integral solution of the Helmholtz equation in domains with non-smooth boundaries. IMA J Numer Anal 1993;13:43-66.

[19] Seznec R. Diffraction of sound around barriers: use of the boundary elements technique. J Sound Vibr 1980;73(2):195-209.

[20] Amini S, Kirkup SM. Solution of Helmholtz equation in the exterior domain by elementary boundary integral methods. J Comput Phys 1995;118(2):208-21.

[21] Burton AJ. The solution of Helmholtz' equation in exterior domains using integral equations. National Physical Laboratory; 1973.

[22] Burton AJ, Miller GF. The application of integral equation methods to the numerical solution of some exterior boundary-value problems. Proc R Soc A-Math Phys Eng Sci 1971;323(1553):201-10.

23] Sladek V, Sladek J, Tanaka M. Optimal transformations of the integration variables in computation of singular integrals in BEM. Int J Numer Methods Eng 2000;47(7):1263-83.

[24] Tanaka M, Sladek V, Sladek J. Regularization techniques applied to boundary element methods. Appl Mech Rev 1994;47(10):457-99.

[25] Guiggiani M. Formulation and numerical treatment of boundary integra equations with hypersingular kernels. In: Sladek V, Sladek J, editors. Singular integrals in boundary element methods. Southampton: Computational Mechanics Publications; 1998. p. 85-124.

[26] Chen JT, Hong HK. Review of dual boundary element methods with emphasis on hypersingular integrals and divergent series. Appl Mech Rev 1999;52(1):17-33.

[27] Schenck HA. Improved integral formulation for acoustic radiation problems. J Acoust Soc Am 1968;44(1):41-58.

[28] Seybert AF, Soenarko B, Rizzo FJ, Shippy DJ. A special integral equation formulation for acoustic radiation and scattering for axisymmetric bodies and boundary conditions. J Acoust Soc Am 1986;80(4):1241-7.

[29] Chen IL, Chen JT, Liang MT. Analytical study and numerical experiments for radiation and scattering problems using the CHIEF method. J Sound Vibr $2001 ; 248(5): 809-28$

[30] Juhl P. A numerical study of the coefficient matrix of the boundary element method near characteristic frequencies. J Sound Vibr 1994;175(1):39-50.

[31] Chen JT, Chen IL, Chen KH. Treatment of rank deficiency in acoustics using SVD. J Comput Acoust 2006;14(2):157-84.

[32] Wu TW, Seybert AF. A weighted residual formulation for the CHIEF method in acoustics. J Acoust Soc Am 1991;90(3):1608-14

[33] Segalman DJ, Lobitz DW. A method to overcome computational difficulties in the exterior acoustics problem. J Acoust Soc Am 1992;91(4):1855-61.

[34] Lee L, Wu TW. An enhanced CHIEF method for steady-state elastodynamics. Eng Anal Boundary Elem 1993:12(2):75-83.

[35] Mohsen A, Hesham M. An efficient method for solving the nonuniqueness problem in acoustic scattering. Commun Numer Methods Eng 2006;22(11): 1067-1076.

[36] Okubo T, Fujiwara K. Efficiency of a noise barrier on the ground with an acoustically soft cylindrical edge. J Sound Vibr 1998;216(5):771-90.

37] Fujiwara K, Hothersall DC, Kim C. Noise barriers with reactive surfaces. Appl Acoust 1998;53(4):255-72.

[38] Kiusalaas J. Numerical methods in engineering with Matlab. Cambridge: Cambridge University Press; 2005.

39] Seybert AF, Rengarajan TK. The use of CHIEF to obtain unique solution for acoustic radiation using boundary integral equations. J Acoust Soc Am 1987;81(5):1299-306

[40] Sladek V, Sladek J. Introductory notes on singular integrals. In: Sladek V, Sladek J, editors. Singular integrals in boundary element methods. Southampton: Computational Mechanics Publications; 1998. p. 1-29.

[41] Singh KM, Tanaka M. Analytical integration of weakly singular integrals in boundary element analysis of Helmholtz and advection-diffusion equations. Comput Methods Appl Mech Eng 2000;189(2):625-40. 
[42] Sladek V, Sladek J, Tanaka M. Numerical integration of logarithmic and nearly logarithmic singularity in BEMs. Appl Math Model 2001;25:901-22.

[43] Singh KM, Tanaka M. On non-linear transformations for accurate numerical evaluation of weakly singular boundary integrals. Int J Numer Methods Eng 2001;50(8):2007-30.

[44] Tsamasphyros G, Theotokoglou EE. A quadrature formula for integrals with nearby singularities. Int J Numer Methods Eng 2006;67(8):1082-93.

[45] Telles JCF. A self-adaptive co-ordinate transformation for efficient numerical evaluation of general boundary element integrals. Int J Numer Methods Eng 1987;24:959-73.

[46] Sato M, Yoshiyoka S, Tsukui K, Yuuki R. Accurate numerical integration of singular kernals in the two-dimensional boundary element method. In: Brebbia CA, editor. Boundary elements X. Berlin: Springer; 1988. p. 279-96.

[47] Telles JCF, Oliveira RF. Third degree polynomial transformation for boundary element integrals: further improvements. Eng Anal Boundary Elem 1994;13: 135-141.

[48] Doblaré M, Gracia L. On non-linear transformations for the integration of weakly-singular and Cauchy principal value integrals. Int J Numer Methods Eng 1997;40(18):3325-58.

[49] Johnston PR, Elliott D. A generalisation of Telles' method for evaluating weakly singular boundary element integrals. J Comput Appl Math 2001; 131(1-2):223-41
[50] Elliott D, Johnston PR. Gauss-Legendre quadrature for the evaluation of integrals involving the Hankel function. J Comput Appl Math 2008;211(1): 23-35.

[51] Johnston PR, Johnston BM. A simple device to improve the accuracy of evaluating weakly singular boundary element integrals. Commun Numer Methods Eng 2002;18(3):189-94.

[52] Johnston PR, Elliott D. Transformations for evaluating singular boundary element integrals. J Comput Appl Math 2002;146(2):231-51.

[53] Johnston PR. Application of sigmoidal transformations to weakly singular and near-singular boundary element integrals. Int J Numer Methods Eng 1999;45(10):1333-48.

[54] Ma H, Kamiya N. Distance transformation for the numerical evaluation of near singular boundary integrals with various kernels in boundary element method. Eng Anal Boundary Elem 2002;26(4):329-39.

[55] Johnston PR, Elliott D. A sinh transformation for evaluating nearly singular boundary element integrals. Int J Numer Methods Eng 2005;62(4):564-78.

[56] Johnston BM, Johnston PR, Elliott D. A sinh transformation for evaluating twodimensional nearly singular boundary element integrals. Int J Numer Methods Eng 2007;69(7):1460-79.

[57] Morse PM, Ingard KU. Theoretical acoustics. New York: McGraw-Hill; 1968.

[58] Treeby BE, Pan J, Paurobally RM. Acoustic scattering by a sphere with a hemispherically split boundary condition. J Acoust Soc Am 2007;122(1):46-57. 